\title{
Proximity effect in a superconductor-topological insulator heterostructure based on first principles
}

\author{
Kyungwha Park $\odot,{ }^{1, *}$ Gabor Csire, ${ }^{2, \dagger}$ and Balazs Ujfalussy $\oplus^{3, \ddagger}$ \\ ${ }^{1}$ Department of Physics, Virginia Tech, Blacksburg, Virginia 24061, USA \\ ${ }^{2}$ Catalan Institute of Nanoscience and Nanotechnology (ICN2), CSIC, BIST, Campus UAB, 08193 Bellaterra, Barcelona, Spain \\ ${ }^{3}$ Wigner Research Centre for Physics, P.O. Box 49, H-1525 Budapest, Hungary
}

(Received 5 May 2020; revised 22 July 2020; accepted 31 August 2020; published 15 October 2020)

\begin{abstract}
Superconductor-topological insulator (SC-TI) heterostructures were proposed to be a possible platform to realize and control Majorana zero modes. Despite experimental signatures indicating their existence, univocal interpretation of the observed features demands theories including realistic electronic structures. To achieve this, we solve the Kohn-Sham-Dirac-Bogoliubov-de Gennes equations for ultrathin $\mathrm{Bi}_{2} \mathrm{Se}_{3}$ films on superconductor palladium telluride within the fully relativistic Korringa-Kohn-Rostoker method and investigate quasiparticle spectra as a function of chemical potential and film thickness. We find multiple proximity-induced gaps where the gap sizes highly depend on characteristics of the TI states. The TI Dirac interface state is relevant to the induced gap only when the chemical potential is close to the Dirac-point energy. Otherwise, at a given chemical potential, the largest induced gap arises from the highest-energy quantum-well states, whereas the smallest gap arises from the TI topological surface state with its gap size depending on the TI pairing potential.
\end{abstract}

DOI: 10.1103/PhysRevB.102.134504

\section{INTRODUCTION}

Superconducting proximity effects in nanoscale heterostructures have been studied on various experimental platforms since proposals [1-3] on the realization of Majorana zero modes in them. Representative systems are superconductor-topological insulator (SC-TI) heterostructures [4-11] and semiconductor nanowires or ferromagnetic chains on SC substrates [12-19]. Majorana zero modes remain robust over local environmental perturbations due to the topological nature, which allows their applications to quantum computation [20]. Experimental data have exhibited signatures of Majorana zero modes, yet there remains debate about whether other possibilities can be completely excluded for them. This doubt is raised by inconsistent experimental findings and current theoretical limitations.

In SC-TI heterostructures, it was predicted that the topological TI interface state creates proximity-induced topological superconductivity where the edge state or the vortex lattice can host Majorana zero modes. Keeping this in mind, experiments were performed on $\mathrm{Bi}_{2} \mathrm{Se}_{3}(111)$ or $\mathrm{Bi}_{2} \mathrm{Te}_{3}(111)$ films $(<10 \mathrm{~nm})$ grown on $\mathrm{NbSe}_{2}$ or $\mathrm{Nb}$ substrates, using scanning tunneling microscopy/spectroscopy $[5,7,9]$ and angle-resolved photoemission spectra (ARPES) $[6,10]$. A proximity-induced gap was measured when the Fermi level lies in the TI conduction band. The proximity-induced gap was shown to decrease with increasing TI film thickness. However, both characteristics of the gap and its dependence on film thickness remain elusive and inconsistent among different groups. The induced surface gap was reported to reach $15 \%-40 \%$ of the bulk SC gap $[5,7,10]$ even for thick TI films

\footnotetext{
*Corresponding author: kyungwha@vt.edu

† gabor.csire@icn2.cat

‡ujfalussy.balazs@wigner.mta.hu
}

where the interface and top-surface Dirac states do not hybridize. Intriguingly, transport experiments on $\mathrm{Bi}_{2} \mathrm{Se}_{3}$ with a $\mathrm{Pb}$ overlayer showed zero resistivity and a proximity-induced gap about $1 \mu \mathrm{m}$ away from the interface [4].

Theoretical efforts have been made to understand the proximity effect in SC-TI heterostructures, using the Bogoliubovde Gennes Hamiltonian for SC and effective models for TI [2,3,21-24]. The TI model Hamiltonian ranges from the Fu-Kane-Mele model [25] to the $k \cdot p$ model [26] with parameter values from calculated band structures using densityfunctional theory (DFT). For a chemical potential away from the Dirac point, triplet pairing was predicted [2,21], and proximity-induced pairing types were classified based on symmetries [23]. Despite this success, the current theoretical approaches have many limitations due to the lack of information on realistic band structures and interface effects as well as insufficient treatment of quasi-two-dimensional experimental systems. The reported theoretical studies either neglected quantum-well states (QWSs) or included generic QWSs, although the experimental Fermi level typically lies in the TI conduction band, and they oversimplified the Fermi surfaces despite their importance in the induced gap features.

We investigate quasiparticle spectra of heterostructures composed of thin $\mathrm{Bi}_{2} \mathrm{Se}_{3}$ overlayers on a $s$-wave $\mathrm{SC}$ palladium telluride (PdTe) substrate by solving the Dirac-Bogoliubovde Gennes (DBdG) equations [27] using the fully relativistic screened Korringa-Kohn-Rostoker (SKKR) Green's function method $[28,29]$ within DFT. We calculate the band structure of Andreev bound states and determine proximity-induced gap features in the TI layers, considering two different TI pairing potentials, as the overlayer thickness and chemical potential are varied. Several different types of induced gaps appear depending on the characteristics of the TI states for a given overlayer thickness and chemical potential. The induced gap arising from the interface TI Dirac state is the 


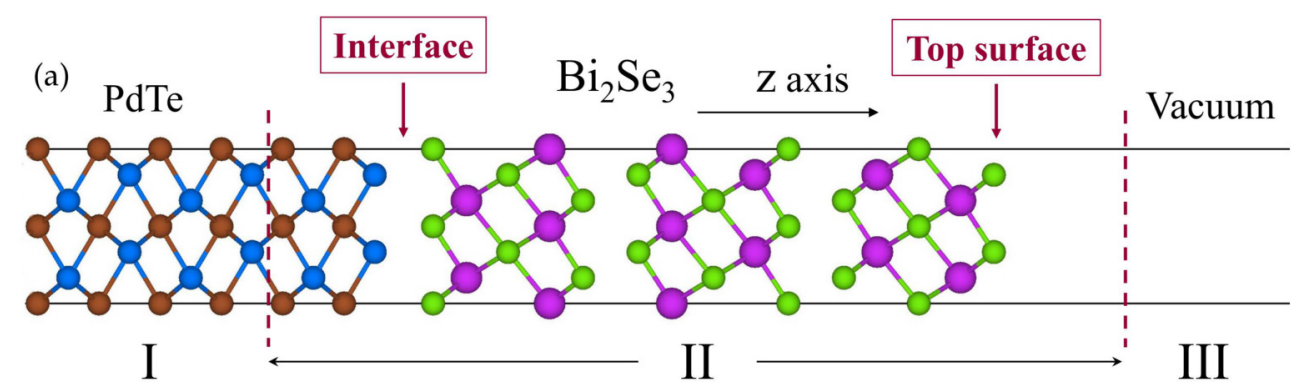

(b) BSF bulk PdTe

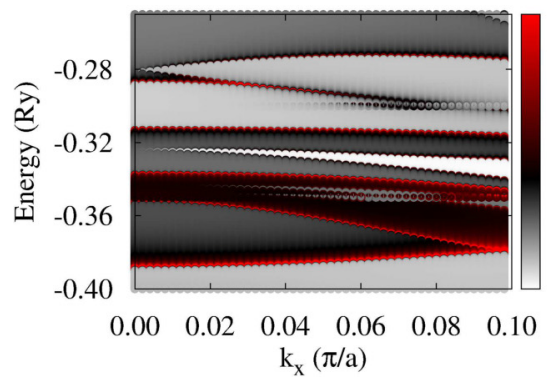

(c)

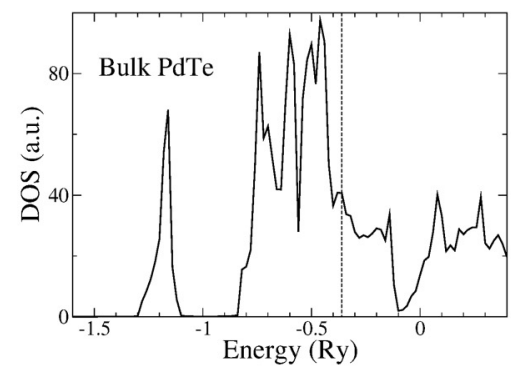

(d) Semi-infinite $\mathrm{Bi}_{2} \mathrm{Se}_{3}$

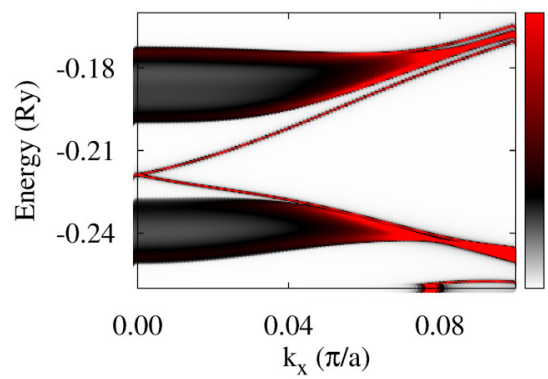

FIG. 1. (a) Schematic diagram of the SC-TI heterostructures for our SKKR-based simulations. A $\mathrm{Bi}_{2} \mathrm{Se}_{3}(111)$ film is overlaid on PdTe(001). Region II (interface region) consists of $\mathrm{Bi}_{2} \mathrm{Se}_{3}$ films of 1-6 QLs (about 1-6 nm) and four PdTe atomic layers, while regions I and III are semi-infinite PdTe and vacuum layers, respectively. The color code is as follows: Pd (brown), Te (blue), Se (green), Bi (purple). (b) SKKR-calculated BSF contours and (c) DOS for bulk PdTe (dashed vertical line: $E_{F}$ ) and (d) SKKR-calculated BSF contours for a semi-infinite $\mathrm{Bi}_{2} \mathrm{Se}_{3}$ system. In (b) and (d) the BSF weight increases from white to red in vertical color bars. Dirac surface states are shown within the bulk band gap in (d). In the SC-TI heterostructures (Fig. 2) the TI Dirac point is found near -0.29 Ry due to a shift of the Madelung potential.

largest, while the gap from the top-surface TI state is the smallest, independent of the thickness, chemical potential, and TI pairing potential. The induced gap associated with a given QWS type increases as the chemical potential decreases. The induced gap sizes do not have a spatial dependence except for the top-surface TI Dirac state for thick TI films. The induced gap size from the top-surface TI Dirac state is highly susceptible to the TI pairing potential, although that is not the case for the induced gap from the interface TI Dirac state.

We first discuss our systems of interest in Sec. II and present our first-principles calculations of the electronic structure of the heterostructures in the normal state as a function of overlayer thickness in Sec. III A. We then show our calculated spectral functions of the heterostructures in the SC state with two different TI pairing potentials as a function of overlayer thickness and chemical potential in Sec. III B. We make a brief comparison of our results with relevant experimental data in Sec. IIIC and make a conclusion in Sec. IV.

\section{SYSTEMS OF INTEREST}

To incorporate realistic electronic structures in the superconducting proximity effect, we simulate $\mathrm{Bi}_{2} \mathrm{Se}_{3}$ films of one to six quintuple layers (QLs) overlaid on SC PdTe [Fig. 1(a)] within the fully relativistic SKKR method [28,29]. We consider such heterostructures due to the following advantages: (i) the good lattice match at the interface, (ii) the reasonable SC transition temperature of PdTe, (iii) the single Dirac cone of $\mathrm{Bi}_{2} \mathrm{Se}_{3}$ at a given surface [26], and (iv) experimental data on $\mathrm{SC}-\mathrm{Bi}_{2} \mathrm{Se}_{3}$ heterostructures (despite different $\mathrm{SC}$ substrates) $[4-7,10]$. Bulk $\mathrm{Bi}_{2} \mathrm{Se}_{3}$ has a rhombohedral structure (space group 166, $R \overline{3} \mathrm{~m}$ ) with experimental lattice constants $a=4.143 \AA$ and $c=28.636 \AA[30]$. As shown in Fig. 1, Se-Bi-Se-Bi-Se atomic layers form 1 QL (about $1 \mathrm{~nm}$ ) along the [111] direction, and individual QLs are bonded via weak van der Waals interaction. The (111) surface has a hexagonal in-plane lattice with a lattice constant $a=4.143 \AA$. Bulk PdTe has a NiAs-type hexagonal structure (space group 194, $P 6_{3} / m m c$ ) with experimental lattice constants $a=4.152 \AA$ and $c=5.671 \AA$ [31]. When a Se-terminated $\mathrm{Bi}_{2} \mathrm{Se}_{3}(111)$ film is interfaced with a Te-terminated $\mathrm{PdTe}(001)$ substrate, the in-plane lattice mismatch between $\mathrm{Bi}_{2} \mathrm{Se}_{3}$ and $\mathrm{PdTe}$ is about $0.2 \%$. PdTe is a type-II SC with a critical temperature of $4.5 \mathrm{~K}$ [31], and its SC gap $\Delta_{\mathrm{PdTe}}^{\exp }$ is about $0.71 \mathrm{meV}$ $(=0.05 \mathrm{mRy})$ at zero temperature [31]. The electron-phonon coupling of PdTe is 1.4 [31], whereas the reported electronphonon coupling of $\mathrm{Bi}_{2} \mathrm{Se}_{3}$ has an upper bound of 0.43 [32].

As required in the SKKR formalism, the SC-TI heterostructures are divided into three regions, I-III [Fig. 1(a)]. Regions I and III are semi-infinite PdTe and vacuum layers, respectively. Region II consists of Pd-Te-Pd-Te atomic layers overlaid with $\mathrm{Bi}_{2} \mathrm{Se}_{3}$ layers (1-6 QLs) and four to six vacuum layers on top. The heterostructures have twodimensional translational symmetry with the lattice constant of $\mathrm{Bi}_{2} \mathrm{Se}_{3}, 4.143 \AA$. Our choice of the in-plane lattice constant 
(a) $\mathrm{Bi}_{2} \mathrm{Se}_{3} 1 \mathrm{QL}$ on $\mathrm{PdTe}$

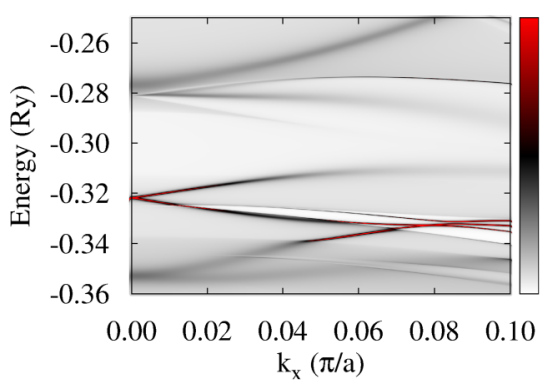

(d) $\mathrm{Bi}_{2} \mathrm{Se}_{3} 4 \mathrm{QLs}$ on PdTe

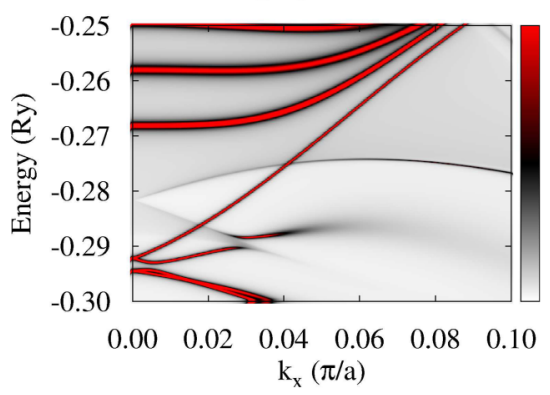

(g) 5QLs/PdTe Topmost QL

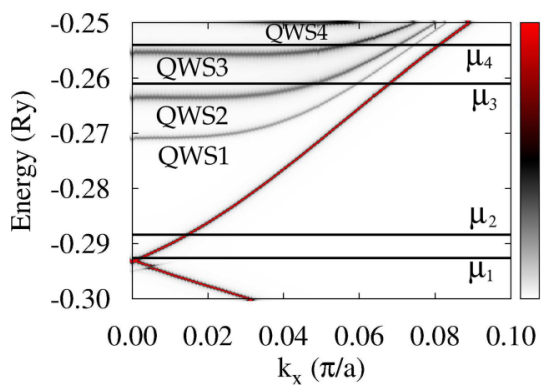

(b) $\mathrm{Bi}_{2} \mathrm{Se}_{3} 2 \mathrm{QLs}$ on PdTe

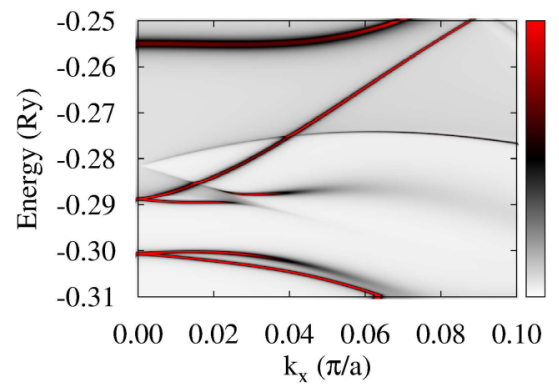

(e) $\mathrm{Bi}_{2} \mathrm{Se}_{3} 5 \mathrm{QLs}$ on PdTe

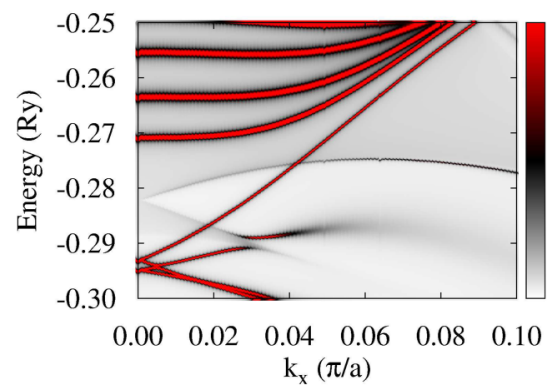

(h) 5QLs/PdTe Interface QL

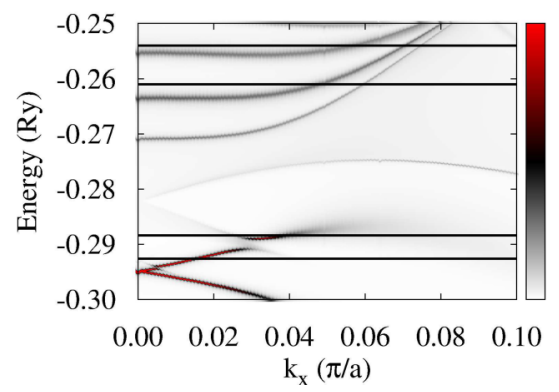

(c) $\mathrm{Bi}_{2} \mathrm{Se}_{3} 3 \mathrm{QLs}$ on PdTe

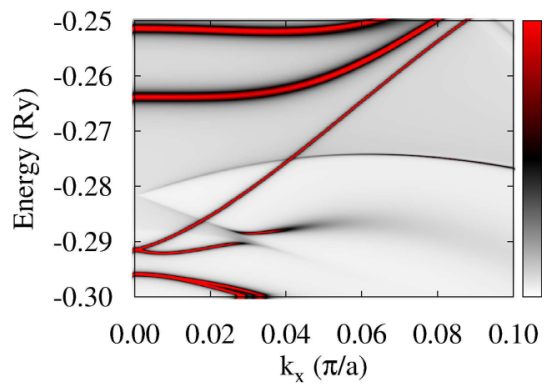

(f) $\mathrm{Bi}_{2} \mathrm{Se}_{3} 6 \mathrm{QLs}$ on PdTe

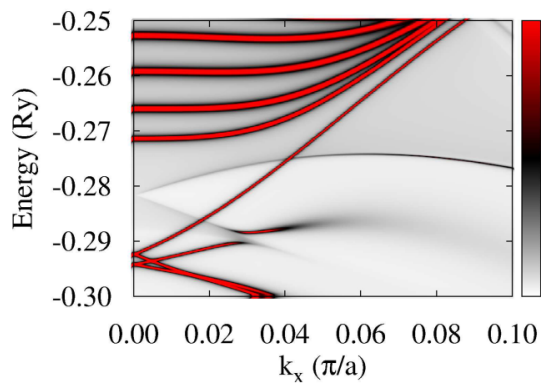

(i) $5 \mathrm{QLs} / \mathrm{PdTe}$ PdTe layers

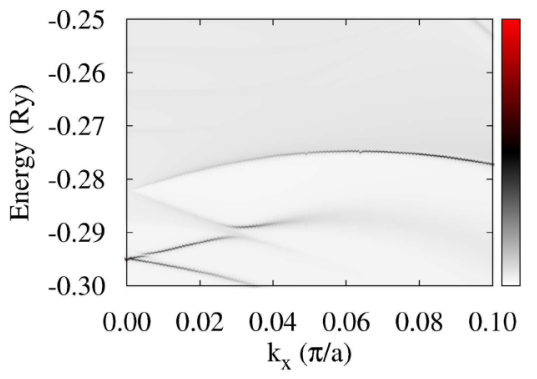

FIG. 2. (a)-(f) Total BSF contours of the $\mathrm{Bi}_{2} \mathrm{Se}_{3}$ overlayers (1-6 QLs) on normal-state PdTe as a function of energy and $k_{x}$. BSF contours of ( $\mathrm{g}$ ) the topmost QL, (h) the interface QL, and (i) the PdTe layers for the 5-QL TI overlayer on normal-state PdTe. The broken inversion symmetry separates the top-surface Dirac states from the interface Dirac states. The surface-hybridization-induced gap appears near $\Gamma$ for (a)-(d). The four horizontal lines in (g) and (h) indicate chemical potential values: $\mu_{1}=-0.2926 \mathrm{Ry}, \mu_{2}=-0.2884 \mathrm{Ry}, \mu_{3}=-0.2610 \mathrm{Ry}$, and $\mu_{4}=-0.2540$ Ry. In (g) QWS1, QWS2, QWS3, and QWS4 are four QWSs.

is made to avoid an effect of strain on the topological surface states $[33,34]$. The out-of-plane lattice constant for PdTe is slightly expanded to conserve the volume of PdTe. Otherwise, experimental lattice constants are used. We consider the band structure only along the $\Gamma-K$ direction, the $k_{x}$ axis, in this work.

\section{RESULTS AND DISCUSSION}

In multiple-scattering theory the band structure is obtained from site-dependent Bloch spectral functions (BSFs) $A_{i}^{\mathrm{B}}\left(\mathcal{E}, \mathbf{k}_{\|}\right)$, where $i$ denotes the $i$ th atomic site and $\mathcal{E}_{n}\left(\mathbf{k}_{\|}\right)$is the $n$th band energy at in-plane momentum $\mathbf{k}_{\|}$. We calculate the BSF for the $i$ th site (located at $\mathbf{r}_{i}$ ) from its retarded Green's function $G_{i}^{+}\left(\mathcal{E}, \mathbf{r}_{i}, \mathbf{k}_{\|}\right)$:

$$
A_{i}^{\mathrm{B}}\left(\mathcal{E}, \mathbf{k}_{\|}\right)=-\frac{1}{\pi} \operatorname{Im} \operatorname{Tr} \int d \mathbf{r}_{i} G_{i}^{+}\left(\mathcal{E}, \mathbf{r}_{i}, \mathbf{k}_{\|}\right),
$$

where the integration is carried out over a Wigner-Seitz sphere around the atomic site within the atomic sphere approxima- tion. The density of states (DOS) within the SKKR method is obtained from an integral of the BSF over $\mathbf{k}_{\|}$, i.e., $\mathcal{D}(\mathcal{E})=$ $\sum_{i} \int d \mathbf{k}_{\|} A_{i}^{\mathrm{B}}\left(\mathcal{E}, \mathbf{k}_{\|}\right)$. A detailed description of the solution of the DBdG equations within the fully relativistic SKKR method can be found in Refs. [28,29]. A brief method description and computational details, including parameter values for our simulations, are shown in Notes 1 and 2 in the Supplemental Material (SM) [35], respectively.

\section{A. Electronic structure of normal state}

First of all, we present calculated electronic structures of $\mathrm{PdTe}$ and $\mathrm{Bi}_{2} \mathrm{Se}_{3}$ in the normal state separately. Figures 1(b) and 1(c) show BSF contours and the DOS of bulk PdTe, respectively. The calculated $\mathrm{BSF}$ shows a metallic nature. There is a wide range of high electron density below the Fermi level $E_{\mathrm{F}}(-0.36 \mathrm{Ry})$ with a sharp DOS peak around -1.20 Ry which arises from Te $s$ and $p$ orbitals. Figure 1(d) shows BSF contours of a semi-infinite $\mathrm{Bi}_{2} \mathrm{Se}_{3}$ system. We find Dirac surface states within a bulk band gap of about 
(a) Interface Se layer

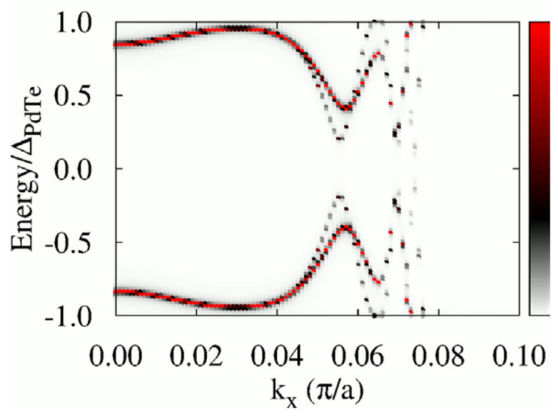

(d) Topmost Se layer

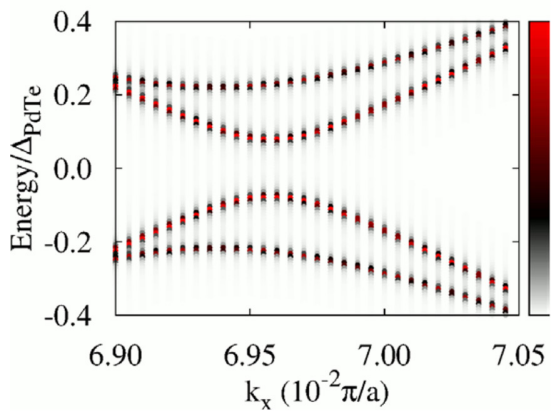

(g) Interface Se layer (electron)

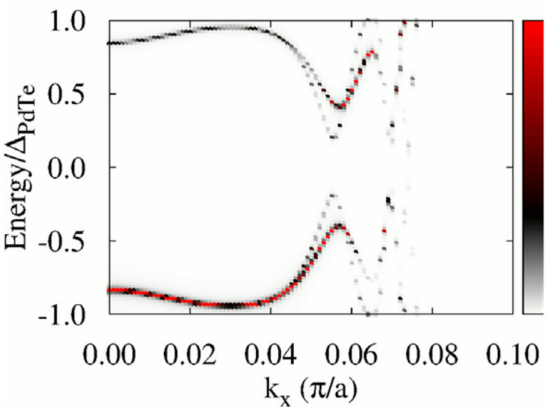

(j) Interface Se layer (hole)

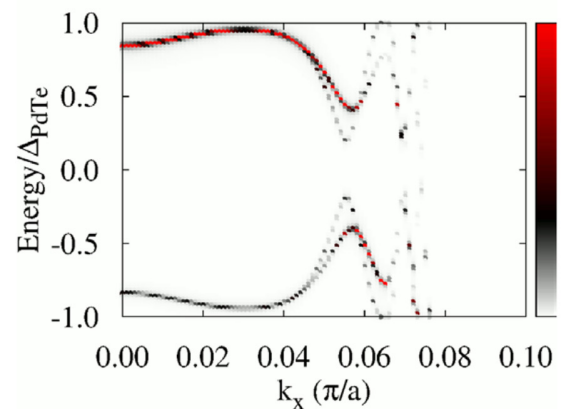

(b) Middle Se layer

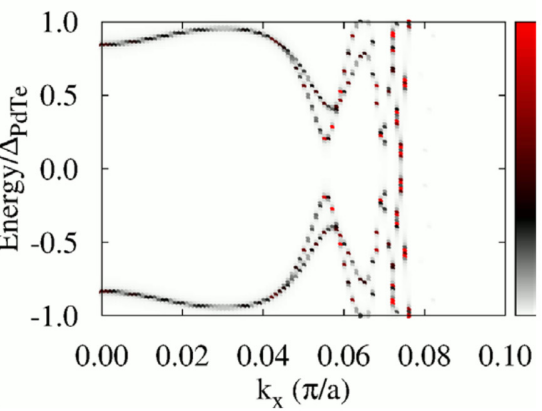

(e) Topmost Se layer

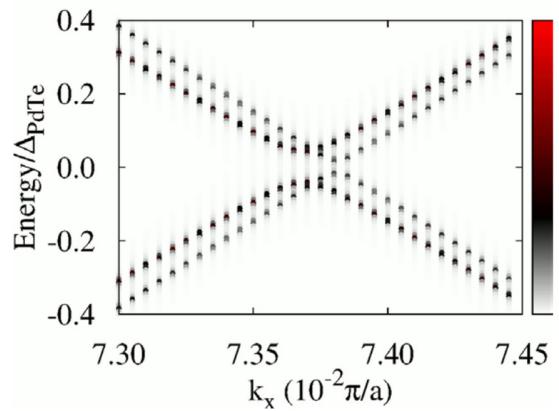

(h) Topmost Se layer (electron)

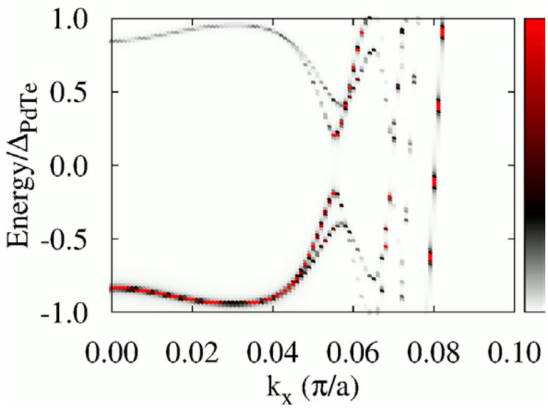

(k) Topmost Se layer (hole)

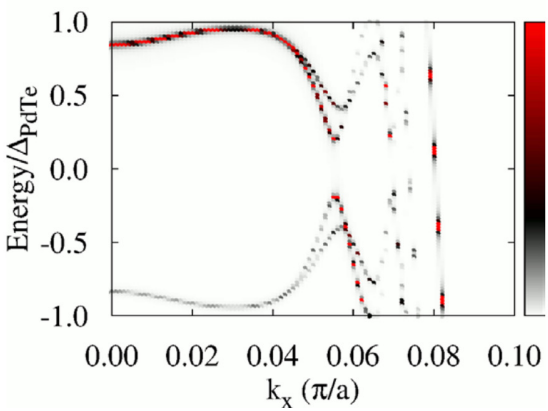

(c) Topmost Se layer

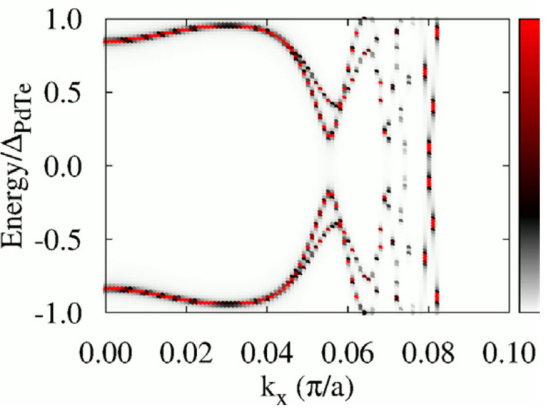

(f) Topmost Se layer

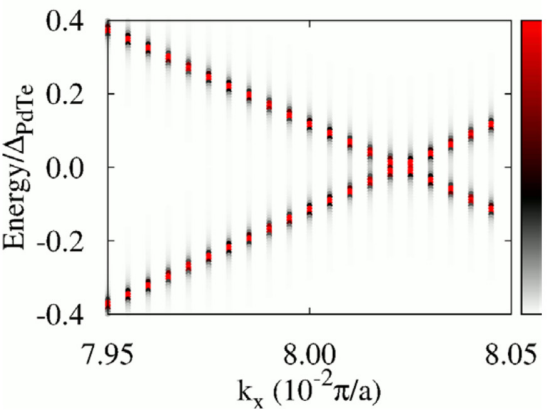

(i) Topmost Se layer (electron)

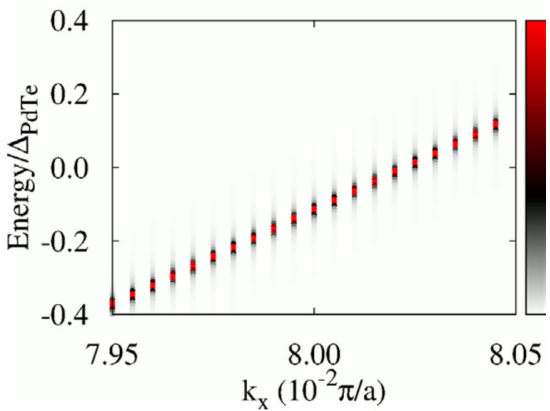

(1) Topmost Se layer (hole)

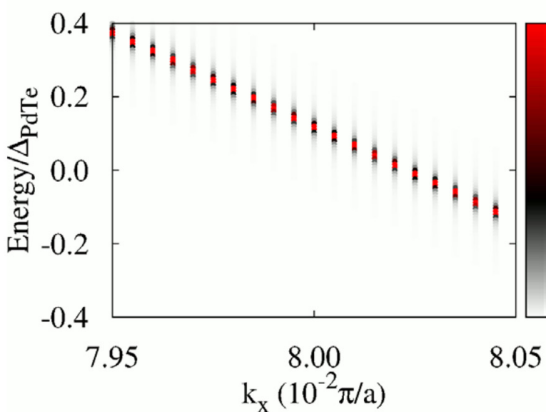

FIG. 3. BSF contours of the 5-QL overlayer on SC PdTe at $\mu_{4}=-0.2540$ Ry, where the energy is in units of the bulk SC gap $\Delta_{\text {PdTe }}(1$ mRy in our calculation). (a) Interface Se, (b) middle Se, and (c) topmost Se layer BSFs. (d)-(f) Zoom-in of (c) near the induced gaps $\Delta_{\mathbf{k} 3}, \Delta_{\mathbf{k} 4}$, $\Delta_{\mathbf{k} 5}, \Delta_{\mathbf{k} 6}$, and $\Delta_{\mathbf{k} 7}$. In (a)-(f) both electron and hole contributions are included. (g) and (j) Electron and hole BSF contours of (a). (h) and (k) Electron and hole BSF contours of (c). (i) and (l) Electron and hole BSF contours of (f). The smallest induced gap $\Delta_{\mathbf{k} 7}$ is less than $0.1 \%$ of the bulk SC gap, as shown in (f).

$0.03 \mathrm{Ry}$ as well as continuous conduction and valence band regions. For $\mathrm{Bi}_{2} \mathrm{Se}_{3}$ slabs, all bands are doubly degenerate due to time-reversal and inversion symmetries, and several QWSs appear in the conduction and valence band regions. For an $N$-QL slab, $N-1(N-2)$ QWSs appear in the conduction (valence) band region [36], as shown in Figs. S1(b) and 1(d) in the SM [35]. For $\mathrm{Bi}_{2} \mathrm{Se}_{3}$ slabs thinner than 5-6 QLs, the top and bottom surface states hybridize, opening an energy gap in the Dirac surface states [36,37]. This gap is referred to as a surface-hybridization gap. The SKKR-calculated band 
structures of PdTe and $\mathrm{Bi}_{2} \mathrm{Se}_{3}$ agree with those using the VASP code [38,39] (Fig. S1 in the SM [35]).

Using the above converged potentials of PdTe and $\mathrm{Bi}_{2} \mathrm{Se}_{3}$, we perform fully relativistic SKKR calculations on the $\mathrm{Bi}_{2} \mathrm{Se}_{3}-\mathrm{PdTe}$ heterostructure in the normal state. The Fermi level of the heterostructure is the same as that of the bulk PdTe. Figures 2(a)-2(f) show calculated the normal-state BSF of the heterostructure with the TI film thickness varying from 1 to $6 \mathrm{QLs}$, where all layers are summed. The gray continuous spectrum in the BSF is similar to that of PdTe [compare Figs. 2(a)-2(f) with Fig. 1(b) or 2(i)]. We find that a shift of the Madelung potential lowers the TI Dirac point around -0.29 Ry and that the top-surface and interface Dirac states are shifted from each other with strong modification of the dispersion of the interface Dirac states. The slope of the dispersion near the Dirac point is substantially reduced, and the states lose the interface-state character somewhat away from $\Gamma$ [see Figs. 2(g) and 2(h)]. The top-surface (interface) Dirac states are identified as states with a large BSF weight onto the topmost (interface) QL. Strong hybridization of the interface states with the substrate causes the strong modification of their dispersion. Similar effects have been reported in various heterostructures involving $\mathrm{Bi}_{2} \mathrm{Se}_{3}[40,41]$. For thin films ( $<5$ QLs) we also observe an energy gap in the vicinity of the two Dirac points which decreases with increasing TI overlayer thickness. This gap is induced by the hybridization between the interface and top-surface Dirac states. The shape and number of TI QWSs, however, remain unchanged with the substrate, but they are quite broadened compared to the Dirac states, as shown in Figs. 2(b)-2(f). This broadening may be caused by scattering of electrons from the substrate. A calculated BSF with finer resolution suggests that each broad QWS peak in Fig. 2 consists of two bands or states. For the 5-QL overlayer on PdTe [Fig. 2(g)], chemical potential $\mu_{4}=-0.2540$ Ry crosses three QWSs (labeled QWS1, QWS2, QWS3) and the top-surface Dirac state, while chemical potential $\mu_{2}=-0.2844$ Ry crosses only the top-surface and interface Dirac states. For thinner TI overlayers, chemical potential $\mu_{4}$ crosses fewer QWSs compared to the 5-QL overlayer. See Figs. S2(b), S1(e), and S1(f) for the characteristics of the QWSs.

\section{B. Electronic structure of the superconducting state}

\section{Induced spectral gap with $\Delta_{\mathrm{eff}}^{\mathrm{TI}}=0$}

Let us now consider that PdTe is in the SC state with a bulk $\mathrm{SC}$ gap $\Delta_{\mathrm{PdTe}}$ of $1.0 \mathrm{mRy}$ and that the TI pairing potential $\Delta_{\text {eff }}^{\mathrm{TI}}$ is zero. We then calculate BSFs of the heterostructures at a fixed chemical potential value. Our choice of the SC gap size of PdTe $\Delta_{\text {PdTe }}$ is about one order of magnitude greater than the experimental value [31] because of our numerical accuracy $(\mu \mathrm{Ry})$. Despite this difference, our result can still be applied to experimental systems as long as the ratio of the induced SC gap to the bulk SC gap is compared with experiment. Thus, we present our calculated induced SC gap in units of $\Delta_{\text {PdTe }}$. In our work, we vary the chemical potential value without doping and compute BSFs in the SC state. See the SM [35], Note 2, for the detailed procedure and justification of our method.

First, we present our result for chemical potential $\mu_{4}=$ $-0.254 \mathrm{Ry}$ (about $520 \mathrm{meV}$ above the Dirac point), which is
TABLE I. Induced SC gaps $\Delta_{\mathbf{k i}}$ in units of the bulk SC gap $\Delta_{\text {PdTe }}$ (=1 mRy in our calculation) at $k_{i}$ and characteristics for the 5-QL overlayer on SC PdTe at chemical potential $\mu_{4}=-0.2540$ Ry. Due to the numerical accuracy, any gap size less than $0.1 \%$ of $\Delta_{\mathrm{PdTe}}$ $(<1.0 \mu \mathrm{Ry})$ is set to zero. The TI pairing potential is set to zero. See Fig. 3. SS denotes the top-surface TI Dirac state.

\begin{tabular}{lllllllc}
\hline \hline & $i=1$ & $i=2$ & $i=3$ & $i=4$ & $i=5$ & $i=6$ & $i=7$ \\
\hline$\Delta_{\mathbf{k i}}\left(\right.$ in $\left.\Delta_{\text {PdTe }}\right)$ & 0.20 & 0.28 & 0.22 & 0.08 & 0.04 & 0.02 & 0 \\
$k_{i}(0.01 \pi / a)$ & 5.550 & 5.700 & 6.930 & 6.960 & 7.370 & 7.380 & 8.023 \\
Origin & QWS3 & QWS3 & QWS2 & QWS2 & QWS1 & QWS1 & SS \\
\hline \hline
\end{tabular}

similar to the experimental Fermi level of SC-TI heterostructures $[6,10]$. Figure 3 shows BSF contours of the 5-QL TI overlayer on SC PdTe as a function of energy and $k_{x}$ at $\mu_{4}=-0.254 \mathrm{Ry}$. Within the bulk SC gap, we find multiple proximity-induced gaps $\Delta_{\mathbf{k i}}$ originating from multiple bands crossing a given chemical potential, where $i$ is the band index. In particular, we focus on the seven induced gap sizes listed in Table I. Two large gaps, $\Delta_{\mathbf{k} 1}$ and $\Delta_{\mathbf{k} 2}$, are clearly seen in Figs. 3(a)-3(c). Gap sizes $\Delta_{\mathbf{k} 3}, \Delta_{\mathbf{k} 4}, \Delta_{\mathbf{k} 5}, \Delta_{\mathbf{k} 6}$, and $\Delta_{\mathbf{k} 7}$ are zoomed in in Figs. 3(d)-3(f). Except for $\Delta_{\mathbf{k} 7}$, all six gap sizes are observed in the BSFs of all TI layers. For an example, see Fig. 4(c) in the case of $\Delta_{\mathbf{k} \mathbf{1}}$. Thus, these gap sizes do not depend on $z$ for a fixed TI-overlayer thickness. The induced gap size is larger with smaller $k_{x}$. The dispersion near the six gap sizes arises from both electron and hole contributions [see Figs. 3(g), 3(h) 3(j), 3(k), S3, and S4]. In the case of $\Delta_{\mathbf{k} 7}$, however, the dispersion appears only for the topmost QL, and it exhibits a positive (negative) slope only from the electron (hole) contribution [see Figs. 3(i) and 3(1)].

The dispersion near the first six induced gaps suggests that Cooper pairs of PdTe tunnel into the TI region, giving rise to the proximity-induced gaps and Andreev bound states. The number and size of gaps can be strongly modified with the interface type and the band structure of non-SC. By comparing the SC-state BSF to the normal-state BSF, we identify that $\Delta_{\mathbf{k} 1}$ and $\Delta_{\mathbf{k} 2}$ originate from Cooper-pair tunneling into QWS3 and that $\Delta_{\mathbf{k} 3}$ and $\Delta_{\mathbf{k} 4}$ originate from QWS2. Similarly, $\Delta_{\mathbf{k} 5}$ and $\Delta_{\mathbf{k} 6}$ arise from Cooper-pair tunneling into QWS1, and $\Delta_{\mathbf{k} 7}$ arises from the top-surface Dirac state (see also Fig. S5). To elucidate the origin of the two different gap sizes from each QWS, we plot normalized SC-state and normal-state $\mathrm{BSF}$ at fixed energies and $k_{x}$ points near the gap size $\Delta_{\mathbf{k} \mathbf{1}}$. As shown in Fig. 4(a), the two SC-state BSF qualitatively differ from each other, while the two normal-state BSF are indistinguishable. The state with a larger spectral weight near the interface gives rise to a larger induced gap. Figure 4(c) also clearly shows the two induced gaps at $k_{1}$. This two-gap feature is consistent with that of the normal-state BSF (Fig. S6). The induced gap size overall increases as Cooper pairs tunnel into higher-energy QWS because of stronger coupling with the substrate. See Fig. 4(b) and Table I. The dispersion near $\Delta_{\mathbf{k} 7}$ [Figs. 3(i) and 3(1)] is quite distinct from that near the other gaps. Cooper pairs do not seem to efficiently tunnel into the top-surface Dirac state which is strongly localized at the top surface, giving rise to zero induced gap.

We now investigate the effect of chemical potential on the proximity-induced gap at the 5-QL overlayer by considering 

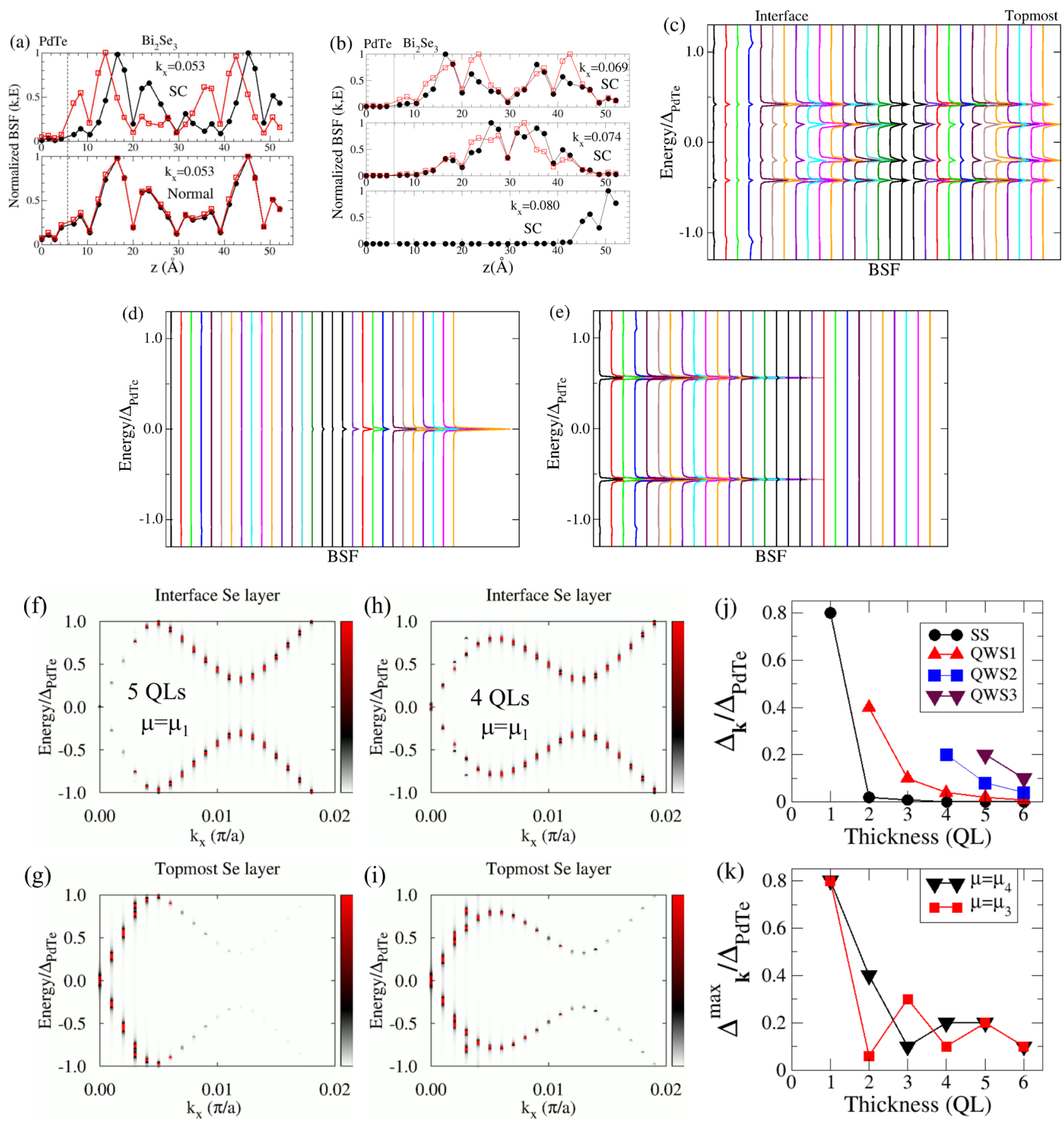

FIG. 4. (a) Normalized SC-state and normal-state BSFs vs $z$ at fixed energies and $k_{x}$ (near $k_{1}$ ). The black (red) curve here corresponds to the black (red) BSF peak at energy of $-0.32 \Delta_{\text {PdTe }}\left(-0.52 \Delta_{\text {PdTe }}\right)$ in Fig. 3(a). (b) Normalized SC-state BSFs vs $z$ at fixed $k_{x}$ and energies with $\mu_{4}$ near $\Delta_{\mathbf{k} 3}, \Delta_{\mathbf{k} 4}, \Delta_{\mathbf{k} 5}, \Delta_{\mathbf{k} 6}$, and $\Delta_{\mathbf{k} 7}$. (c)-(e) Layer-resolved SC-state BSFs vs energy at $k_{1}$ with $\mu_{4}$ (Table I) and at $k_{x}=0.01369 \pi / a$ and $0.03700 \pi / a$ with $\mu_{2}$ for 5-QL/PdTe, respectively. The BSF of each layer is shifted and scaled. (f)-(i) BSF contours of the 5-QL and 4-QL overlayers with $\mu_{1}$, respectively. (j) Induced gap from different states vs thickness at $\mu_{4}$. (k) $\Delta_{\mathbf{k}}^{\max } / \Delta_{\text {PdTe }}$ vs thickness at $\mu_{3}$ and $\mu_{4}$. See the main text for the definition of $\Delta_{\mathbf{k}}^{\max }$. The energy is in units of the SC gap of bulk PdTe $\Delta_{\text {PdTe }}$.

$\mu_{3}, \mu_{2}$, and $\mu_{1}$, as indicated in Fig. 2(g). We consider a wide range of the chemical potential such as $0.038 \mathrm{Ry}(=0.52 \mathrm{eV})$ since the Fermi level of experimental SC-TI heterostructures can be varied by doping, TI film thickness, or growth conditions from well above the bottom of the TI conduction band to the TI Dirac point, which is about $0.5 \mathrm{eV}[5-7,9,10]$. For $\mu_{3}$ five distinct gap sizes are found. Similarly to the case of $\mu_{4}$, the smallest gap is zero from the top-surface Dirac state, while the rest four gaps are as significant as those for $\mu_{4}$. The dispersion near the four gaps has characteristics of Andreev states. As chemical potential decreases, the number of gaps decreases, whereas the gap size associated with a given QWS type (such as QWS1, QWS2) increases. See Fig. S7. For $\mu_{2}$ and $\mu_{1}$, only two gap sizes are observed, and Andreev states exist only near the larger gap. Figures 4(d) and 4(e) show layer-resolved BSF with $\mu_{2}$, and Figs. 4(f) and 4(g) show 

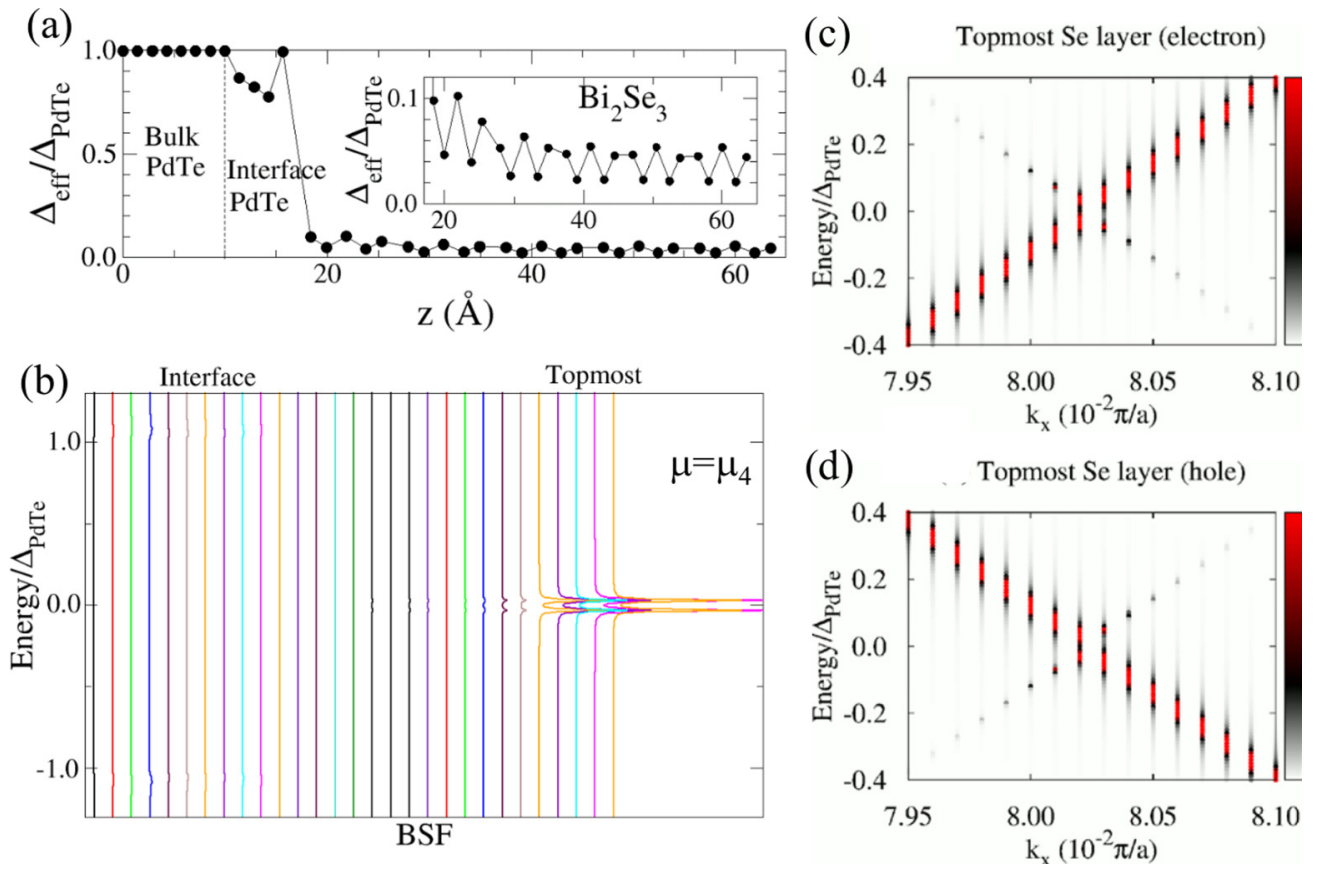

FIG. 5. (a) Self-consistently calculated effective pairing potential vs $z$ for the 5-QL/PdTe with the TI region zoomed in. (b) Layer-resolved BSF for the 5-QL/PdTe at $k_{x}=k_{4}$ with $\mu_{4}$. (c) and (d) Electron and hole BSFs of the topmost Se layer corresponding to (b). In (b)-(d) the pairing potential in (a) is used. The energy is in units of the SC gap of bulk PdTe $\Delta_{\text {PdTe }}$.

BSF of the interface and topmost layers with $\mu_{1}$. The two gaps appear only closer to the interface QL or the topmost QL. The highly suppressed slope of the dispersion does not allow Cooper pairs to tunnel into the interface TI state until chemical potential reaches near the Dirac points. For $\mu_{2}$ and $\mu_{1}$, only top-surface and interface TI states are involved. The induced gap from the interface TI state is largest among the gaps found.

To examine the effect of TI film thickness, we plot the smaller induced gap from each TI-state type (i.e., QWS1, QWS2, QWS3, interface or top surface) vs thickness at $\mu_{4}$ [Fig. 4(j)]. We find that the induced gap from higher-energy QWSs decays much more slowly than that from lower-energy QWSs and that the gap from the top-surface Dirac state becomes zero for thickness greater than 3 QLs. Figure 4(k) shows the "maximum" gap from the gap sizes shown in Fig. 4(j) as a function of overlayer thickness at $\mu_{3}$ and $\mu_{4}$. Interestingly, the maximum gap oscillates with thickness. For overlayers thinner than 5 QLs, the surface-hybridization effect is also seen in the induced gap. The gap size from the top-surface Dirac state becomes noticeable, and the induced gap sizes do not depend on $z$. Compare Figs. 4(h) and 4(i) with Figs. 4(f) and 4(g) for $\mu_{1}$. Tables in the SM [35] list all induced gap sizes.

\section{Induced spectral gap with $\Delta_{\text {eff }}^{\mathrm{TI}} \neq 0$}

We study an effect of the TI pairing potential $\Delta_{\text {eff }}^{\mathrm{TI}}$ by keeping $\Delta_{\mathrm{PdTe}}$ the same as before and considering that $\Delta_{\text {eff }}^{\mathrm{TI}} \neq 0$. Although electron-phonon coupling of the TI cannot induce superconductivity itself, it can create a finite pairing potential induced by the superconductor [2]. Figure 5(a) shows the effective pairing potential $\Delta_{\text {eff }}$ calculated self-consistently for the 5-QL overlayer on PdTe using semiphenomenological parameters within the SKKR method. See the SM [35] for the detailed procedure. As shown in the inset, the pairing potential decays very slowly in the TI region. With this calculated pairing potential, we obtain BSFs for the 5-QL overlayer on SC PdTe at $\mu_{4}$. As listed in Table II, the induced gap from the top-surface Dirac state becomes noticeable with Andreevstate characteristics [Figs. 5(b)-5(d)], whereas the gap from high-energy QWSs does not change much compared to the case of zero TI pairing potential. We expect that topological edge states within the smallest induced gap can trap Majorana zero modes with broken time-reversal symmetry $[2,3]$ and that pairing type of the proximity-induced superconductivity may be identified from gap anisotropy [3] and/or unique spinorbital textures of the Andreev states [42].

TABLE II. Induced spectral gaps (in units of the bulk SC gap, $\Delta_{\mathrm{PdTe}}=1 \mathrm{mRy}$ ) for the 5-QL overlayer on SC PdTe at chemical potential $\mu_{4}=-0.2540$ Ry with two different TI pairing potentials $\Delta_{\text {eff }}^{\mathrm{TI}}$. A smaller gap is chosen when two gaps are found at $k_{x}$ values close to each other. QWS1, QWS2, QWS3, and SS denote the firstlowest, second, and third QWSs in the TI conduction band region and the top-surface TI Dirac state, respectively. Due to the numerical accuracy, any gap size $<\mu \mathrm{Ry}$ is set to zero.

\begin{tabular}{lcc}
\hline \hline & $\Delta_{\text {eff }}^{\mathrm{TI}} \neq 0[$ Fig. 5(a) $]$ & $\Delta_{\text {eff }}^{\mathrm{TI}}=0$ \\
\hline QWS3 & 0.220 & 0.200 \\
QWS2 & 0.110 & 0.080 \\
QWS1 & 0.056 & 0.016 \\
SS & 0.030 & 0 \\
\hline \hline
\end{tabular}




\section{Comparison with experiment}

Let us make a brief qualitative comparison of our results with experimental and model-Hamiltonian studies, considering that our SC substrate differs from those used in experiments. References [6,10] showed momentum-resolved proximity-induced gaps using ARPES. In Ref. [6] the proximity-induced gap from the bulk TI state significantly differs from the gap from the TI surface state, while in Ref. [10] that is not the case. Our results are consistent with the former experimental data whether $\Delta_{\text {eff }}^{\mathrm{TI}}$ is set to zero or not. The discrepancy may originate from the experimental difficulty in identifying the peak center of the spectral function corresponding to the bulk and surface states. We find that an accurate estimate of the induced gap from the TI surface state requires high precision in the momentum such as $10^{-5} \pi / a$. With a precision of $0.001 \pi / a$, the induced gap can be overestimated by two orders of magnitude. However, the induced gap from bulk states is insensitive to the precision of the momentum. Comparing our results to scanning tunneling microscopy experimental data [5,7], we obtain underestimated induced gaps even for $\Delta_{\text {eff }}^{\mathrm{TI}} \neq 0$. Model-Hamiltonian studies predicted the zero gap from the top-surface TI state for thick TI overlayers [21,24], which is consistent with our result. However, the rich features of the induced gaps that we find were not obtained from the model-Hamiltonian approaches.

\section{CONCLUSION}

In summary, we simulated $\mathrm{Bi}_{2} \mathrm{Se}_{3}$ films of 1-6 nm overlaid on SC PdTe by solving the DBdG equations with two pairing potential profiles within the SKKR method, finding that multiple proximity-induced SC gaps arise from the unique TI band structure and its modifications under the SC substrate. The size of the induced gap strongly varies with characteristics of TI states which are partially occupied at the Fermi level. Cooper pairs tunnel into higher-energy QWSs more efficiently, and the induced gap from higher-energy QWSs decreases more slowly with increasing TI film thickness. For thick TI films, the induced gap from the top-surface Dirac state becomes zero with zero TI pairing potential, whereas the gap can be substantial for finite TI pairing potential. For a given thick TI film, the induced gap from the interface Dirac state appears only near the interface QL, although the induced gap size from each QWS does not depend on $z$. Our findings demonstrate the importance of the consideration of the realistic TI band structure for studies of the superconducting proximity effect, and they can be used for future larger-scale simulations and experimental studies of topological edge states or nanowires on SC substrates in pursuit of Majorana zero modes.

\section{ACKNOWLEDGMENTS}

We are grateful to P. Rakyta for setting up the initial $\mathrm{Bi}_{2} \mathrm{Se}_{3}$ structures. The computational support was provided by San Diego Supercomputer Center under Grant No. DMR060009N and Virginia Tech Advanced Research Computing in the United States. B.U. was supported by the Hungarian National Research, Development and Innovation Office under Contract No. K131938 and BME Nanotechnology FIKP grants. G.C. acknowledges support from the European Union's Horizon 2020 research and innovation program under Marie Sklodowska-Curie Grant Agreement No. 754510, Spanish MINECO (the Severo Ochoa Centers of Excellence Program under Grant No. SEV-2017-0706), Spanish MICIU, AEI, EU FEDER (Grant No. PGC2018-096955-B-C43), Generalitat de Catalunya (Grant No. 2017SGR1506 and the CERCA Program), and the European Union MaX Center of Excellence (EU-H2020 Grant No. 824143).
[1] A. Y. Kitaev, Phys.-Usp. 44, 131 (2001).

[2] L. Fu and C. L. Kane, Phys. Rev. Lett. 100, 096407 (2008).

[3] J. Alicea, Rep. Prog. Phys. 75, 076501 (2012).

[4] F. Yang, F. Qu, J. Shen, Y. Ding, J. Chen, Z. Ji, G. Liu, J. Fan, C. Yang, L. Fu, and L. Lu, Phys. Rev. B 86, 134504 (2012)

[5] M.-X. Wang, C. Liu, J.-P. Xu, F. Yang, L. Miao, M.-Y. Yao, C. L. Gao, C. Shen, X. Ma, X. Chen, Z.-A. Xu, Y. Liu, S.-C. Zhang, D. Qian, J.-F. Jia, and Q.-K. Xue, Science 336, 52 (2012).

[6] S.-Y. Xu, N. Alidoust, I. Belopolski, A. Richardella, C. Liu, M. Neupane, G. Bian, S.-H. Huang, R. Sankar, C. Fang, B. Dellabetta, W. Dai, Q. Li, M. J. Gilbert, F. Chou, N. Samarth, and M. Z. Hasan, Nat. Phys. 10, 943 (2014).

[7] J.-P. Xu, C. Liu, M.-X. Wang, J. Ge, Z.-L. Liu, X. Yang, Y. Chen, Y. Liu, Z.-A. Xu, C.-L. Gao, D. Qian, F.-C. Zhang, and J.-F. Jia, Phys. Rev. Lett. 112, 217001 (2014).

[8] Q. L. He, H. Liu, M. He, Y. H. Lai, H. He, G. Wang, K. T. Law, R. Lortz, J. Wang, and I. K. Sou, Nat. Commun. 5, 4247 (2014).

[9] J.-P. Xu, M.-X. Wang, Z. L. Liu, J.-F. Ge, X. Yang, C. Liu, Z. A. Xu, D. Guan, C. L. Gao, D. Qian, Y. Liu, Q.-H. Wang,
F.-C. Zhang, Q.-K. Xue, and J.-F. Jia, Phys. Rev. Lett. 114, 017001 (2015).

[10] D. Flototto, Y. Ota, Y. Bai, C. Zhang, K. Okazaki, A. Tsuzuki, T. Hashimoto, J. N. Eckstein, S. Shin, and T.-C. Chiang, Sci. Adv. 4, eaar7214 (2018).

[11] H.-H. Sun, M.-X. Wang, F. Zhu, G.-Y. Wang, H.-Y. Ma, Z.-A. Xu, Q. Liao, Y. Lu, C.-L. Gao, Y.-Y. Li, C. Liu, D. Qian, D. Guan, and J.-F. Jia, Nano Lett. 17, 3035 (2017).

[12] V. Mourik, K. Zuo, S. M. Frolov, S. R. Plissard, E. P. A. M Bakkers, and L. P. Kouwenhoven, Science 336, 1003 (2012).

[13] O. Gül, H. Zhang, F. K. de Vries, J. van Veen, K. Zuo, V. Mourik, S. Conesa-Boj, M. P. Nowak, D. J. van Woerkom, M. Quintero-PÃrez, M. C. Cassidy, A. Geresdi, S. Koelling, D. Car, S. R. Plissard, E. P. A. M. Bakkers, and L. P. Kouwenhoven, Nano Lett. 17, 2690 (2017).

[14] H. Zhang, C.-X. Liu, S. Gazibegovic, D. Xu, J. A. Logan, G. Wang, N. van Loo, J. D. S. Bommer, M. W. A. de Moor, D. Car, R. L. M. Op het Veld, P. J. van Veldhoven, S. Koelling, M. A. Verheijen, M. Pendharkar, D. J. Pennachio, B. Shojaei, J. S. Lee, C. J. Palmstrøm, E. P. A. M. Bakkers, S. D. Sarma, and L. P. Kouwenhoven, Nature (London) 556, 74 (2018). 
[15] S. Nadj-Perge, I. K. Drozdov, J. Li, H. Chen, S. Jeon, J. Seo, A. H. MacDonald, B. A. Bernevig, and A. Yazdani, Science 346, 602 (2014).

[16] R. Pawlak, M. Kisiel, J. Klinovaja, T. Meier, S. Kawai, T. Glatzel, D. Loss, and E. Meyer, npj Quantum Inf. 2, 16035 (2016).

[17] B. E. Feldman, M. T. Randeria, J. Li, S. Jeon, Y. Xie, Z. Wang, I. K. Drozdov, B. A. Bernevig, and A. Yazdani, Nat. Phys. 13, 286 (2017).

[18] M. Ruby, B. W. Heinrich, Y. Peng, F. von Oppen, and K. J. Franke, Nano Lett. 17, 4473 (2017).

[19] A. Palacio-Morales, E. Mascot, S. Cocklin, H. Kim, S. Rachel, D. K. Morr, and R. Wiesendanger, Sci. Adv. 5, eaav6600 (2019).

[20] A. Kitaev, Ann. Phys. (NY) 303, 2 (2003).

[21] T. D. Stanescu, J. D. Sau, R. M. Lutchyn, and S. Das Sarma, Phys. Rev. B 81, 241310(R) (2010).

[22] M. Lababidi and E. Zhao, Phys. Rev. B 83, 184511 (2011).

[23] A. M. Black-Schaffer and A. V. Balatsky, Phys. Rev. B 87, 220506(R) (2013).

[24] C.-K. Chiu, W. S. Cole, and S. Das Sarma, Phys. Rev. B 94, 125304 (2016).

[25] L. Fu, C. L. Kane, and E. J. Mele, Phys. Rev. Lett. 98, 106803 (2007).

[26] H. Zhang, C.-X. Liu, X.-L. Qi, X. Dai, Z. Fang, and S.-C. Zhang, Nature Phys. 5, 438 (2009).

[27] K. Capelle and E. K. U. Gross, Phys. Rev. B 59, 7140 (1999).

[28] G. Csire, B. Ujfalussy, J. Cserti, and B. Gyorffy, Phys. Rev. B 91, 165142 (2015).

[29] G. Csire, A. Deak, B. Nyari, H. Ebert, J. F. Annett, and B. Ujfalussy, Phys. Rev. B 97, 024514 (2018).

[30] S. Nakajima, J. Phys. Chem. Solids 24, 479 (1963).

[31] A. B. Karki, D. A. Browne, S. Stadler, J. Li, and R. Jin, J. Phys.: Condens. Matter 24, 055701 (2012).

[32] X. Zhu, L. Santos, C. Howard, R. Sankar, F. C. Chou, C. Chamon, and M. El-Batanouny, Phys. Rev. Lett. 108, 185501 (2012).
[33] S. M. Young, S. Chowdhury, E. J. Walter, E. J. Mele, C. L. Kane, and A. M. Rappe, Phys. Rev. B 84, 085106 (2011).

[34] W. J. Yang, C. W. Lee, D. S. Kim, H. S. Kim, J. H. Kim, H. Y. Choi, Y. J. Choi, J. H. Kim, K. Park, and M.-H. Cho, J. Phys. Chem. C 122, 23739 (2018).

[35] See Supplemental Material at http://link.aps.org/supplemental/ 10.1103/PhysRevB.102.134504 for (i) our formalism, (ii) computational details, (iii) VASP-calculated results in the normal state, (iv) SKKR-calculated characteristics of quantum-well states, (v) tables of proximity-induced SC gap sizes vs film thickness and $\mu$, and (vi) additional SC-state BSFs for the 5-QL overlayer on PdTe, which includes Refs. [43-48].

[36] K. Park, C. De Beule, and B. Partoens, New J. Phys. 15, 113031 (2013).

[37] Y. Zhang, K. He, C.-Z. Chang, C.-L. Song, L.-L. Wang, X. Chen, J.-F. Jia, Z. Fang, X. Dai, W.-Y. Shan, S.-Q. Shen, Q. Niu, X.-L. Qi, S.-C. Zhang, X.-C. Ma, and Q.-K. Xue, Nat. Phys. 6, 584 (2010).

[38] G. Kresse and J. Furthmuller, Phys. Rev. B 54, 11169 (1996).

[39] G. Kresse and J. Furthmuller, Comput. Mater. Sci. 6, 15 (1996).

[40] K. Govaerts, K. Park, C. De Beule, B. Partoens, and D. Lamoen, Phys. Rev. B 90, 155124 (2014).

[41] Y.-T. Hsu, K. Park, and E.-A. Kim, Phys. Rev. B 96, 235433 (2017).

[42] Y. Fukaya, S. Tamura, K. Yada, Y. Tanaka, P. Gentile, and M. Cuoco, Phys. Rev. B 100, 104524 (2019).

[43] D. M. Ceperley and B. J. Alder, Phys. Rev. Lett. 45, 566 (1980).

[44] M. Suvasini and B. Gyorffy, Phys. C (Amsterdam, Neth.) 195, 109 (1992).

[45] G. Csire, S. Schonecker, and B. Ujfalussy, Phys. Rev. B 94, 140502(R) (2016).

[46] G. Csire, J. Cserti, I. Tutto, and B. Ujfalussy, Phys. Rev. B 94, 104511 (2016).

[47] P. Rakyta, B. Ujfalussy, and L. Szunyogh, New J. Phys. 17, 123011 (2015).

[48] P. E. Blöchl, Phys. Rev. B 50, 17953 (1994). 